\title{
Constitutive GFP-UTF1 Expression Interferes with ES and EC Cell Differentiation
}

\author{
Rajkumar P. Thummer, Loes J. Drenth-Diephuis and Bart J.L. Eggen*
}

Department of Neuroscience, Section Medical Physiology, University of Groningen, University Medical Center Groningen, A. Deusinglaan 1, 9713 AV Groningen, The Netherlands

\begin{abstract}
Embryonic stem cells have the ability to self-renew and can differentiate into all cell types of the three embryonic germ lineages. The undifferentiated embryonic cell Transcription Factor 1 (UTF1) gene is highly expressed in ES cells and we previously reported that UTF1 is tightly associated with chromatin and is required for differentiation of pluripotent mouse embryonic stem (ES) and embryonic carcinoma (EC) cells. In this study, we generated ES and EC cell lines constitutively expressing GFP-UTF1 to further investigate its role in differentiation. ES and EC cells constitutively expressing GFP-UTF1were suppressed in their proliferation and were still dependent on LIF for self-renewal. Embryoid body (EB) differentiation of GFP-UTF1 overexpressing ES cells showed both normal differentiation as well as a delayed or incomplete differentiation of a subset of cells. GFP-UTF1 was persistently expressed in undifferentiated cells whereas GFP-UTF1 expression was not detected in differentiated cells. Where GFP-UTF1 expressing ES cells differentiated normally in response to DMSO, EC cell differentiation was completely blocked. When ES and EC cells expressing GFP-UTF1 were treated with RA, differentiation markers were induced and endogenous UTF1 and GFP-UTF1 protein levels decreased. However, GFP-UTF1 and UTF1 (in ES cells) mRNA was still detected indicating that degradation of (GFP-)UTF1 protein preceded down regulation of (GFP-) UTF1 mRNA, suggesting that RA induced UTF1 degradation. Summarizing these data indicate that similar to UTF1 depletion, overexpression of GFP-UTF1 interfered with ES and EC cells differentiation.
\end{abstract}

\section{Keywords: UTF1; ES cells; EC cells; Differentiation; Chromatin}

\section{Introduction}

Embryonic stem (ES) cells are derived from the inner cell mass (ICM) of blastocyst embryos and defined by their self-renewal capacity and pluripotency; the ability to form all ectoderm, mesoderm and endoderm-derived cell types. Several signaling pathways, transcription factors and epigenetic regulators have been implicated in the maintenance of ES cell pluripotency. Signaling pathways involved in ES cell pluripotency include, but are not limited to, the LIF, BMP, mTOR, MAPK and Wnt pathways [1-6]. Additionally, pluripotency is also regulated by a transcription factor network with Oct4, Sox 2 and Nanog as key members [7-10]. In addition to these signaling pathways and transcription factors, there is substantial evidence that epigenetic regulators like the polycomb-group proteins [11], chromatin remodeling complexes like SWI/SNF [12,13], NuRD [14,15] and other chromatin modifying proteins like Jmjd1a and Jmjd2c [16] contribute to the maintenance of ES cell pluripotency [17].

Chromatin in ES cells is in an open and permissive state, globally transcribed and maintained in an open conformation by chromatinremodeling enzymes such as Chd1 [18-20]. Therefore, it is proposed that epigenetic regulation is important to stabilize ES cells pluripotency through minimizing transcriptional fluctuations, while at the same time ensuring a chromatin state that allows for differentiation along different developmental programs [11,17].

Recently, we showed that undifferentiated embryonic cell Transcription Factor 1 (UTF1) is required for differentiation as ES and EC cells with strongly reduced levels of UTF1 were defective in their differentiation [21]. Recently it was reported that UTF1 inhibited neuronal differentiation of $\mathrm{p} 19$ embryonic carcinoma cells and that a loss of UTF1 in these cells facilitated neuronal differentiation [22]. UTF1 is highly expressed in the ICM of blastocysts and is later restricted to the primordial germ cells and spermatogonial stem cells [23-25]. UTF1 is furthermore expressed in germ cell neoplasms, neuroblastomas and
EC cells [26,27]. UTF1 is a stable chromatin-associated transcriptional repressor with core histone-like properties and its expression rapidly decreases upon differentiation of both ES and EC cells [21,23,28,29]. The purpose of this study was to determine the effect of constitutive GFP-UTF1 expression on ES and EC cell differentiation, as well as on ES cell LIF dependency.

\section{Materials and Methods}

\section{Constructs, cell culture and transfections}

pPyCAG-cHA-IP-GFP-HA-mUTF1 was generated by transferring eGFP-HA-UTF1 from pcDNA3-eGFP-HA-mUTF1 [21] to pPyCAGcHA-IP (kindly provided by H. Niwa).

IB10 ES cells were grown on $0.1 \%$ gelatin-coated dishes in buffalo rat liver cell-conditioned medium supplemented with $1,000 \mathrm{U} / \mathrm{ml}$ leukemia inhibitory factor (LIF; Chemicon), nonessential amino acids, $100 \mathrm{U} / \mathrm{ml}$ penicillin, $100 \mu \mathrm{g} / \mathrm{ml}$ streptomycin (Invitrogen) and $0.1 \mathrm{mM} 2$-mercaptoethanol at $37^{\circ} \mathrm{C}$ and $5 \% \mathrm{CO}_{2}$. P19CL6 EC cells [30] were grown in a-MEM (Invitrogen) supplemented with $10 \%$ fetal bovine serum (FBS) and $100 \mathrm{U} / \mathrm{ml}$ penicillin, $100 \mu \mathrm{g} / \mathrm{ml}$ streptomycin (Invitrogen) at $37^{\circ} \mathrm{C}$ and $5 \% \mathrm{CO}_{2}$.

*Corresponding author: Bart J.L. Eggen, Department of Neuroscience, Section Medical Physiology, University of Groningen, University Medical Center Groningen, A. Deusinglaan 1, 9713 AV Groningen, The Netherlands, Tel: +31-50-3632136; Fax: +31-50-3632751; E-mail: b.j.l.eggen@umcg.nl

Received July 24, 2012; Accepted September 11, 2012; Published September 14,2012

Citation: Thummer RP, Drenth-Diephuis LJ, Eggen BJL (2012) Constitutive GFPUTF1 Expression Interferes with ES and EC Cell Differentiation. J Stem Cell Res Ther 2:127. doi:10.4172/2157-7633.1000127

Copyright: (C) 2012 Thummer RP, et al. This is an open-access article distributed under the terms of the Creative Commons Attribution License, which permits unrestricted use, distribution, and reproduction in any medium, provided the original author and source are credited. 
To generate GFP-UTF1 overexpressing cell lines, $10^{7}$ ES cells or $7.5 \times 10^{4}$ P19CL6 cells were electroporated with $10 \mu \mathrm{g}$ or transfected with $5 \mu \mathrm{g}$ pPyCAG-cHA-IP-GFP-HA-UTF1 DNA, respectively. Clones were grown in the presence of $2 \mu \mathrm{g} / \mathrm{ml}$ Puromycin and selected based on GFP expression using fluorescence microscopy and western blotting.

\section{Subnuclear fractionation and western blotting}

Subnuclear fractionation was performed as described previously [31]. Fractions were analyzed using an $\alpha \mathrm{UTF} 1$ antibody [21] and a goat anti-rabbit IgG-HRP (1:1000, Pierce).

Western blotting was performed as described previously [21]. Cells were washed with cold PBS and incubated in lysis buffer $(400 \mathrm{mM}$ $\mathrm{NaCl}, 20 \mathrm{mM}$ Tris-HCl, pH 7.8, 1\% NP-40, 0.5\% sodium deoxycholate, $2 \mathrm{mM}$ EDTA, $2 \mathrm{mM}$ DTT, and protease inhibitors) for $30 \mathrm{~min}$ on ice. Next, cell lysates were collected by scraping, sonicated and cleared by centrifugation at $4^{\circ} \mathrm{C}$ and $14,000 \mathrm{rpm}$ for $10 \mathrm{~min}$. Protein concentrations were determined by Bradford assays and equal amounts of protein were resolved by SDS-PAGE and transferred to nitrocellulose membranes.

For western analysis, the following primary antibodies were used: UTF1 [21], GFP (1:2000, Santa Cruz Biotechnology, Inc.), Oct4 (1:1000, H-134; Santa Cruz Biotechnology, Inc.), Troma1 (1:2000, Developmental Studies Hybridoma Bank, USA), GATA4 (1:500, C-20; Santa Cruz Biotechnology, Inc.), Vimentin (1:1000, C-20; Santa Cruz Biotechnology, Inc.), GAP43 (C-19, Santa Cruz Biotechnology, Inc.), GFRa-1 (H-70, Santa Cruz Biotechnology, Inc.) and actin (1:10.000, C4; MP Biomedicals). Secondary antibodies used were goat anti-rabbit IgG-HRP (1:1000, Pierce), goat anti-mouse IgG-HRP (1:2000, Santa Cruz Biotechnology, Inc.) and donkey anti-goat IgG-HRP (1: 2000, Santa Cruz Biotechnology, Inc.).

\section{Cell proliferation}

For proliferation analysis of wt and GFP-UTF1 overexpressing cell lines, $5 \times 10^{4}$ cells were plated in 6-well plates in triplicate. Cells were counted using a haemocytometer after 12, 24, 36, 48 and $60 \mathrm{hr}$ (ES cells) or $8,16,24,32,40,48$ and $56 \mathrm{hr}$ (EC cells).

\section{Embryoid body (EB) formation of ES cells}

For EB formation, ES cells were suspended from the lids of $10 \mathrm{~cm}$ $\emptyset$ petri dishes in $20 \mu \mathrm{l}$ drops $\left(5 \times 10^{4}\right.$ cells $\left./ \mathrm{ml}\right)$. After $48 \mathrm{hr}$, EBs were transferred to bacterial grade petri dishes and cultured in DMEM (BioWhittakar) containing 10\% FBS (PAA), $100 \mathrm{U} / \mathrm{ml}$ penicillin, 100 $\mu \mathrm{g} / \mathrm{ml}$ streptomycin (Invitrogen). Pictures were taken and total RNA was isolated after $0,3,5$ and 10 days.

For EB formation for protein isolation, $4.5 \times 10^{6}$ cells were seeded in $10 \mathrm{~cm} \varnothing$ bacterial grade petri dishes in DMEM (BioWhittakar) containing $10 \%$ FBS (PAA), $100 \mathrm{U} / \mathrm{ml}$ penicillin, $100 \mu \mathrm{g} / \mathrm{ml}$ streptomycin (Invitrogen). Protein lysates were prepared at day $0,2,4$, 6, 8 and 10 and western analyzed.

\section{EB immunohistochemistry and micoscopy}

EBs (7 days) were collected and fixed in $4 \%$ paraformaldehyde (PFA) in PBS for $10 \mathrm{~min}$ at room temperature (rt). Next, EBs were cryoprotected in 10\%, 20\% and 30\% sucrose in PBS for $30 \mathrm{~min}$ in each solution and embedded in Tissue-Tek (Sakura Finetek Europe B.V., Alphen aan de Rijn, The Netherlands). For immunohistochemistry, 16 $\mu \mathrm{m}$ sections were cut using a Leica CM 3050 S cryostat and mounted on poly-L-lysine coated glass slides, dried for $1 \mathrm{hr}$ at $\mathrm{rt}$ and stored at $-80^{\circ} \mathrm{C}$.
Prior to staining, sections were dried in a desiccator for $30 \mathrm{~min}$ at $\mathrm{rt}$ and fixed in 4\% PFA in PBS for 10 min at rt. After preincubation with 5\% normal goat serum (NGS), 3\% FBS in PBS, $0.1 \%$ Triton X-100 for 30 min at rt, samples were incubated overnight with the indicated primary antibodies (for antibody details see section subnuclear fractionations and western blotting) diluted in $1 \%$ NGS, $1 \%$ FBS in PBS, $0.1 \%$ Triton $\mathrm{X}-100$ at $4^{\circ} \mathrm{C}$.

After washing (3 times in PBS), secondary antibodies were diluted in PBS $0.1 \%$ Triton X-100 and incubated at $\mathrm{rt}$ for $2 \mathrm{hr}$. After washing (3 times in PBS), sections were embedded in Vectashield (Vector Laboratories). Fluorescent images were taken using a Zeiss 780 laserscanning microscope (Carl Zeiss MicroImaging, Inc.) equipped with a plan Apochromat $63 \times$ NA 1.4 oil objective. Green signal was detected using a $488 \mathrm{~nm}$ Argon laser line and 500-550 nm detector. The blue and red signals were detected with $405 \mathrm{~nm}$ and $560 \mathrm{~nm}$ as excitation wavelengths using a solid state laser and detected between $440-480 \mathrm{~nm}$ and 580-620 nm, respectively. Confocal images were deconvoluted using Huygens pro 3.3 (SVI, Hilversum, The Netherlands).

\section{RNA isolation and RT-PCR analyses}

Total RNA was extracted with TRIzol (Invitrogen), treated with DNaseI (Fermentas) for $60 \mathrm{~min}$. and reverse transcribed (RevertAid M-MuLV Reverse Transcriptase; Fermentas). Quantitative PCR reactions were carried out using a SYBR supermix (BIORAD) on an ABI7900HT device (Applied Biosystems) with different sets of Q-PCR primers (Table S1). For each gene, measurements were performed in triplicates. GAPDH was used as an internal standard to calculate relative gene expression levels with the $2^{-\Delta \Delta C} \mathrm{~T}$ method [32]. Details of primers for PCR like sequence, cycle numbers and annealing temperatures used can be found in Table S2. PCR products were loaded on $2 \%$ agarose gels.

\section{Differentiation of ES and EC cells}

For DMSO differentiation, $3.65 \times 10^{5}$ cells were seeded in $6 \mathrm{~cm} \varnothing$ petri dishes in culture medium supplemented with $1 \%$ DMSO (SigmaAldrich). Medium was refreshed every 2 days with $1 \%$ DMSO. Pictures were taken and protein lysates were made at day $0,2,5$, and 10 days (ES cells) or after $0,2,4,6,8,10,12$ and 14 days (EC cells).

For RA differentiation $3 \times 10^{5}$ cells were seeded in gelatin coated 10 $\mathrm{cm} \varnothing$ petri dishes with DMEM (BioWhittakar) containing 10\% FBS (PAA), $100 \mathrm{U} / \mathrm{ml}$ penicillin, $100 \mu \mathrm{g} / \mathrm{ml}$ streptomycin (Invitrogen) and $5 \mu \mathrm{M}$ (ES cells) or $1 \mu \mathrm{M}$ (EC cells) RA. Protein lysates were prepared at $0,2,5$ and 10 days (ES cells) or 0, 2, 4 and 8 days (EC cells).

\section{Results}

We previously reported that UTF1 is tightly chromatin-associated transcriptional repressor. In ES cells with strongly reduced UTF1 levels, the expression of $>1200$ genes is significantly increased [28]. Knock down of UTF1 in ES and EC cells resulted in perturbed or blocked differentiation of these cells, respectively [21]. During ES and EC cell differentiation, UTF1 levels decrease rapidly and to determine the effect of constitutive UTF1 expression, we stably expressed GFPUTF1 in P19CL6 EC and IB10 ES cells. We previously reported that the GFP moiety had no discernible effects on UTF1 functionality. GFP-UTF1, like UTF1, repressed reporter gene activity, localized to mitotic chromosomes and fractionated to the tightly chromatin associated protein fraction in subnuclear fractionations [21]. ES and EC clones with GFP-UTF1 expression levels similar to endogenous UTF1 and unaltered Oct4 levels were selected (Figure 1A). Subnuclear 
Citation: Thummer RP, Drenth-Diephuis LJ, Eggen BJL (2012) Constitutive GFP-UTF1 Expression Interferes with ES and EC Cell Differentiation. J Stem Cell Res Ther 2:127. doi:10.4172/2157-7633.1000127

Page 3 of 7

fractionations indicated that also in these cell lines GFP-UTF1, like UTF1, exclusively localized to the ammonium sulfate fraction, containing the strongly DNA-associated proteins (Figure 1B). GFPUTF1 was exclusively localized to the nucleus and chromosomeassociated during mitosis, a distribution very similar to endogenous UTF1 (Figure 1C). Expression profiling of UTF1-depleted ES cells indicated that in the absence of UTF1 many genes are increased in their expression levels [28]. The effect of GFP-UTF1 expression on the expression levels of several UTF1 target genes was determined using quantitative RT-PCR. As a control, UTF1 depleted ES cells were used, and in these cells, UTF1 transcript levels were reduced and Ly6a and Klf4 expression was increased. Expression of GFP-UTF1 resulted in a significant reduction in Dnttip1, Ly6a and Klf4 expression (Figure 1D). These data strongly suggest that GFP-UTF1 binds to the same target
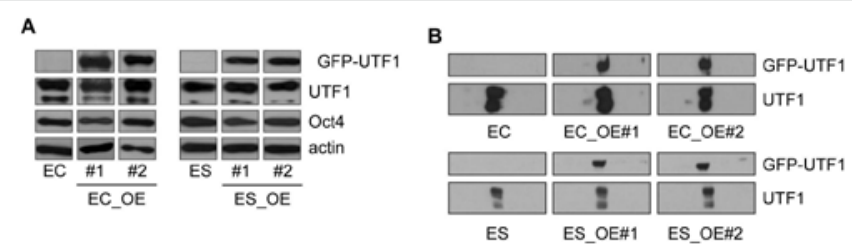

C

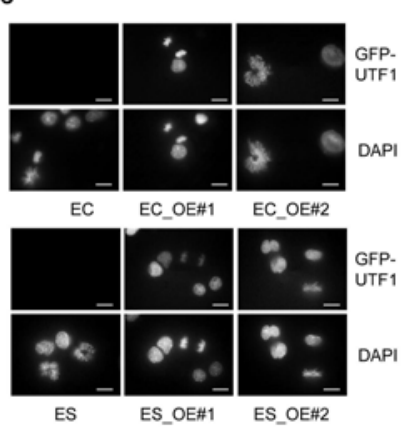

D

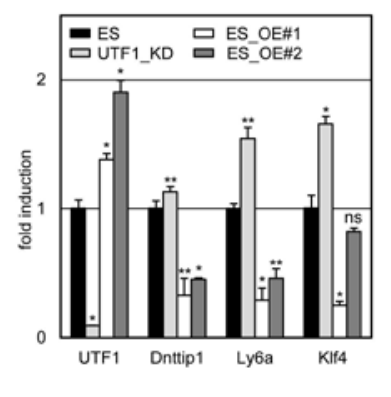

E

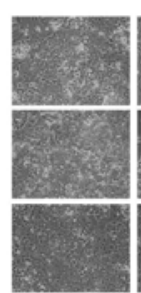

day 0

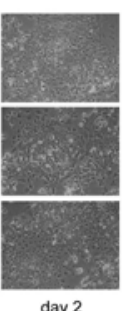

day 2

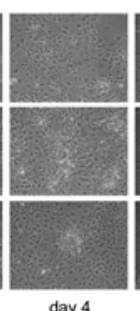

day 4

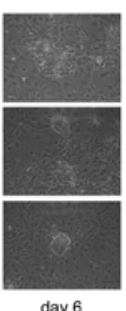

day 6

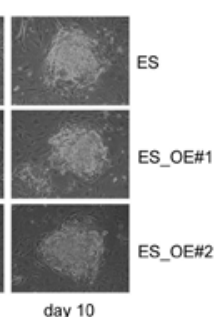

day 10
Figure 1: Characterization of GFP-UTF1 expressing ES and EC cell lines. A) Western analysis of wild type P19CL6 (EC) and IB10 (ES) cells and GFPUTF1 expressing P19CL6 (EC_OE\#1 and \#2) and IB10 (ES OE\#1 and \#2) cells. Cell lysates were analyzed with the antibodies indicated, UTF1 and GFP-UTF1 were both detected with an aUTF1 antibody on the same blot.

B) Subnuclear fractionation of ES and EC cells. Fractions were immunostained with an UTF1 antibody. Fractionation abbreviations: F, free diffusing/ cytoplasmic fraction; D, DNasel released fraction; AS, ammonium sulfate fraction; HS, high salt fraction; $M$, nuclear matrix fraction.

C) Deconvoluted fluorescent images of wild type (ES and EC) and GFP-UTF1 expressing (EC_OE\#1, EC_OE\#2, ES_OE\#1 and ES_OE\#2) ES and EC cell lines. Cells were counterstained with DAPI.

D) Effect of GFP-UTF1 expression on UTF1 target genes. Transcript levels of UTF1 and the indicated UTF1 target genes were determined using quantitative RT-PCR using GAPDH as an internal standard. Mean expression levels and the standard deviations are depicted; *: $p<0.01 ;{ }^{* *}: p<0.05$ and ns: not significant.

E) Phase contrast images of wild type (ES) and GFP-UTF1 expressing (ES OE \#1 and \#2) IB10 ES cells 2-10 days after LIF withdrawal.

\begin{tabular}{|c|c|c|}
\hline cell lines & double time $(\mathrm{hr})$ & fold change \\
\hline EC & $5.84 \pm 0.03^{\star}$ & 1.00 \\
\hline OE\#1 & $5.99 \pm 0.04$ & 1.39 \\
\hline OE\#2 & $6.98 \pm 0.02^{\star}$ & 1.20 \\
\hline OE\#3 & $6.38 \pm 0.09^{\star}$ & 1.09 \\
\hline OE\#4 & $8.13 \pm 0.05^{\star}$ & 1.03 \\
\hline ES & $7.38 \pm 0.11$ & 1.00 \\
\hline OE\#1 & $8.35 \pm 0.15^{\star}$ & 1.13 \\
\hline OE\#2 & $8.13 \pm 0.12^{\star}$ & 1.10 \\
\hline
\end{tabular}

Doubling times of P19CL6 (EC), IB10 (ES) and GFP-UTF1 expressing cell lines (OE\#1-OE\#4), * $p<0.05$

Table 1: ES and EC cell doubling times.

genes as endogenous UTF1. Summarizing, these results indicated that GFP-UTF1, like endogenous UTF1, was restricted to the nucleus, tightly associated with chromatin and repressed expression of known UTF1 target genes, indicating that the GFP moiety had no discernible effect on UTF1 functionality.

UTF1 has been shown to be involved in ES cell proliferation [33] and UTF1 KD ES and EC cells proliferate slower compared to wt cells [21]. Here, the effect of GFP-UTF1 overexpression on ES and EC cell proliferation was determined. A reduced proliferation rate was observed in ES and EC cell lines overexpressing GFP-UTF1 in comparison to wt ES and EC cells (Table 1). For P19CL6 EC cells, an increase in cell cycle time ranging between 3\% (EC_OE\#1) to approximately 39\% (EC_OE\#4) was observed (from $5.8 \mathrm{hr}$ in wt EC cells to $6 \mathrm{hr}$ and 8.1 $\mathrm{hr}$ for lines EC_OE\# 1 and OE\#4, respectively). In ES cells, an increase in cell cycle time of $13 \%$ and $10 \%$ was detected (from $7.4 \mathrm{hr}$ in wt ES cells to $8.4 \mathrm{hr}$ and $8.1 \mathrm{hr}$ for lines ES_OE\#1 and ES_OE\#2 respectively).

Mouse ES cells require LIF for their self-renewal and they spontaneously differentiate in the absence of LIF, although some stem cells persist due to a proposed autocrine/paracrine LIF signaling or by some unknown self-renewal factors [34]. To determine if UTF1 overexpression resulted in LIF independency, GFP-UTF1 expressing ES cells were grown in absence of LIF. Upon LIF withdrawal, morphological changes occurred with similar kinetics in both wt and GFP-UTF1 ES cells, indicating that GFP-UTF1 ES cells were LIF dependent (Figure 1E).

\section{EB differentiation of ES cells overexpressing UTF1}

ES cells with reduced UTF1 levels are perturbed in their capacity to form EBs and failed to differentiate properly [21]. We investigated whether elevated levels of UTF1 also interfered with ES cell differentiation. Embryoid bodies were generated followed by RNA and protein isolation for RT-PCR and western analysis, respectively. GFP-UTF1 overexpressing ES cell lines formed EBs with efficiencies and sizes similar to wt ES cells (Supplementary Figure 1A). At all stages tested, the GFP-UTF1 transgene was still expressed and detected at both the mRNA and protein level (Supplementary Figure 1A and 1B).

RT-PCR analysis of wt and GFP-UTF1 ES lines during EB formation showed similar up regulation of various lineage-specific markers like Brachyury and BMP5 (mesoderm), GATA-4 and GATA-6 (endoderm) and GAP43 and FGF5 (ectoderm) (Supplementary Figure 1B). Expression levels of pluripotency markers Oct-4, REX1 and Nanog decreased in time in wt ES cells but were detected at all time points 
in ES cell lines overexpressing GFP-UTF1, suggesting the presence of an undifferentiated subset of cells (Supplementary Figure 1B). Next, we validated these observations using western analysis. In wt ES cells, UTF1 and Oct 4 protein was not detected after day 4 and differentiation markers were first detected at day 6 (Troma1, GATA4) or day 10 (Vimentin). In GFP-UTF1 expressing cells, UTF1 and Oct4 levels also decreased albeit with slower kinetics then was observed in wt cells. In GFP-UTF1 overexpression lines, induction of differentiation markers was detected at similar time points as in wt cells. These data suggested that GFP-UTF1 overexpressing ES cells were able to differentiate but that a subset of the cells is delayed in their differentiation (Figure 2A).

To determine if a subset of undifferentiated cells expressed GFPUTF1, 7 day embryoid bodies were generated, fixed, sectioned and stained for UTF1, Oct4 and GATA4. Expression of GFP-UTF1 overlapped with Oct4 and UTF1 (Figure 2B), indicating that GFPUTF1 expressing cells still expressed these pluripotency markers. However, when EBs were stained for GATA4 (Figure 2B), GFPUTF1 and GATA4 were expressed in different cells, indicating that these differentiated cells did not express GFP-UTF1. In short, these data suggest that, at least in a subset of cells, GFP-UTF1 expression led to persistent expression of pluripotency markers and perturbed or delayed differentiation.

\section{DMSO differentiation of ES and EC cells}

DMSO initiates a coordinated differentiation program in various cell types. In the presence of DMSO, cells differentiate into cardiac and skeletal muscle along with other mesodermal and endodermal cell types [35]. DMSO-induced differentiation of both wt and GFP-UTF1 expressing ES and EC cells was analyzed. Morphology of both wt and GFP-UTF1 overexpressing ES cells rapidly changed during DMSOinduced differentiation (Figure 3A). Western analysis indicated that
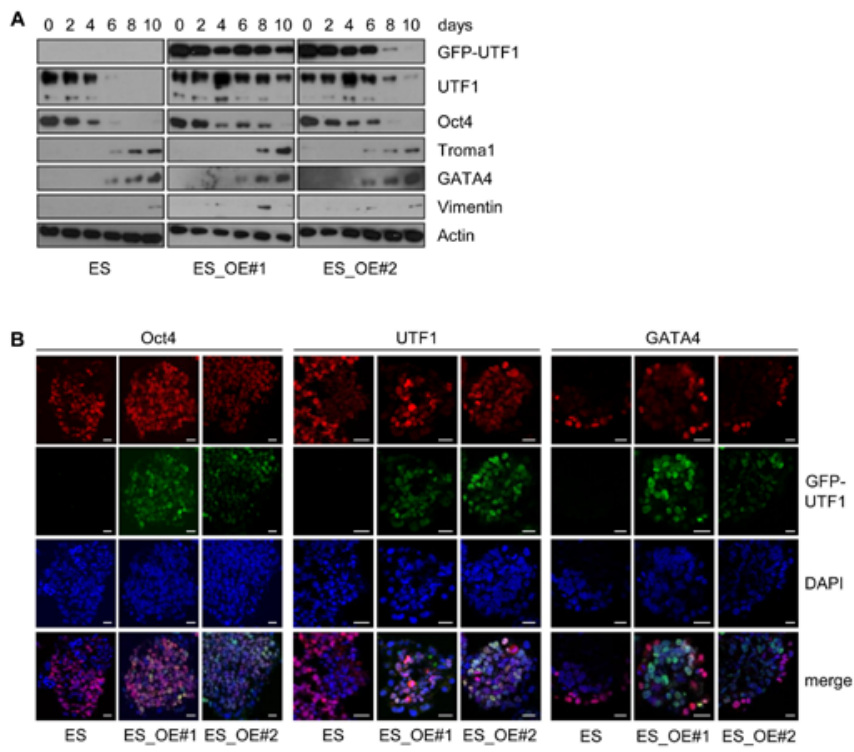

Figure 2: EB differentiation of GFP-UTF1 overexpressing ES cells. A) Western analysis of the expression levels of markers for ES cells (UTF1 and Oct4), mesoderm and endoderm (GATA4), endoderm (Troma1) and mesoderm (Vimentin). Actin levels were determined as a loading control and GFP-UTF1 for transgene expression.

B) Immunohistochemical analysis of wild type (ES) and GFP-UTF1 expressing ES cells (ES_OE\#1 and \#2) using antibodies against Oct4, UTF1 and GATA4 Cells were counterstained with DAPI.
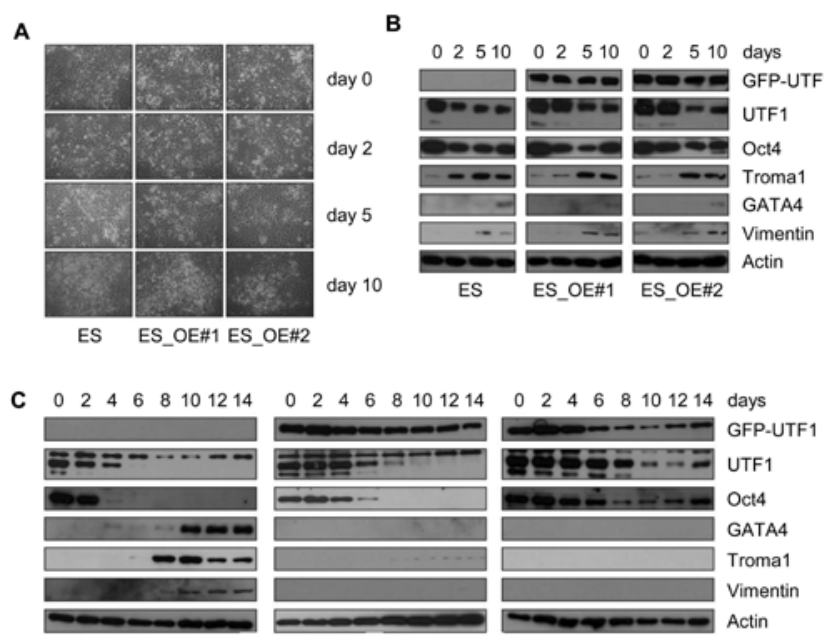

EC

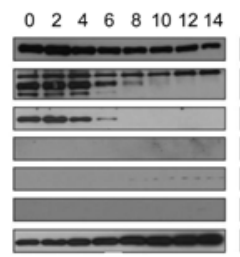

EC_OE\#1

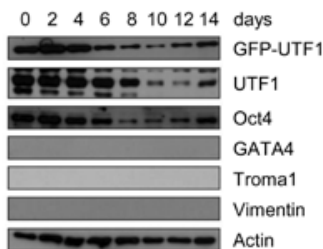

EC_OE\#2
Figure 3: DMSO differentiation of ES and EC cells overexpressing GFPUTF1. A) Phase-contrast images of DMSO-treated (0,2,5 and 10 days) wild type (ES) and GFP-UTF1 overexpressing ES cells (ES_OE\#1 and \#2).

B) Western blots of DMSO-induced differentiation (0,2, 5 and 10 days) of wild type (ES) and GFP-UTF1 overexpressing ES cells (ES_OE\#1 and \#2). Protein lysates were analyzed with antibodies against GFP, UTF1, Oct4, Troma1 (endoderm), GATA4 (endoderm and mesoderm), and Vimentin (mesoderm). Actin staining was performed as a loading control.

C) Western blots of DMSO-induced differentiation of wild type (EC) and GFP-UTF1 overexpressing P19CL6 EC cell lines (EC_OE\#1 and \#2). Protein lysates were collected at the time points indicated and analyzed with antibodies against GFP, UTF1, Oct4, GATA4, Troma1 and Vimentin. Actin staining was performed as a loading control.

GFP-UTF1 overexpressing ES cells differentiated similarly to wild type ES cells, showing increased expression of Troma1, Vimentin and GATA4 (Figure 3B). Expression of the GFP-UTF1 transgene was detected throughout differentiation while expression of endogenous UTF1 decreased during differentiation. Also, Oct 4 expression decreased at day 2 but expression was detected throughout the experiment, suggesting incomplete differentiation of a subset of cells, both in wt and GFP-UTF1 ES cells (Figure 3B).

Next, we investigated the effect of GFP-UTF1 overexpression on the differentiation of P19CL6 EC cells. In wt cells, DMSO induced expression of Troma1 (day 8), Vimentin and GATA4 (day 10); in combination with a complete down regulation of pluripotency markers Oct4 and UTF1. In contrast, GFP-UTF1 overexpressing EC cells failed to differentiate in response to DMSO; Tromal, Vimentin and GATA4 were not or only very faintly detected (Troma1, EC_OE\#1). In EC_OE\#1, endogenous UTF1 was completely down regulated at day 10 and Oct4 expression was not detectable after day 6. In EC_OE\#2, endogenous UTF1 and Oct4 expression was not completely down regulated as expression of both proteins was still observed at day 14 GFP-UTF1 expression was detected at all time points investigated.

Collectively, these data suggest that GFP-UTF1 overexpressing ES cells differentiated normally and that a fraction of the wt and GFP-UTF1 ES cells is refractory to DMSO treatment. In P19CL6 cells overexpressing GFP-UTF1, DMSO-induced differentiation is completely blocked.

\section{RA differentiation of UTF1 overexpressing ES and EC cells}

Retinoic acid (RA) is frequently used to promote ectodermal differentiation of pluripotent cells under a wide variety of culture 
conditions. The morphology of both wt and GFP-UTF1 overexpressing ES cells changed with similar kinetics during RA-induced differentiation (Figure 4A). Immunoblotting showed that in both wt and GFP-UTF1 overexpressing ES cells expression of pluripotency markers Oct 4 and UTF1 decreased rapidly and neural-specific marker GAP43 was first detected at day 5. However, GFP-UTF1 was also undetectable after 5 days of RA treatment (Figure 4B). To determine if RA treatment resulted in silencing of the GFP-UTF1 transgene, RNA was isolated from RA-treated ES cells and analyzed by RT-PCR. Surprisingly, similar levels of GFP-UTF1 transgene expression were detected at all time points tested, indicating that the observed down regulation of GFP-UTF1 after RA treatment was not caused by silencing of the GFPUTF1 transgene (Figure 4C).

RA also induced differentiation of wt and GFP-UTF1 expressing P19CL6 EC cells. After 10 days, Oct4, UTF1 and GFP-UTF1 expression levels were drastically reduced, where GAP43 and GFRa expression was induced (Figure 4D). To determine if the GFP-UTF1 transgene was still expressed, wt and GFP-UTF1 EC cells were treated with RA and RNA was isolated after 0, 2, 4 and 8 days. As was observed in ES cells, equal amounts of the GFP-UTF1 mRNA were detected at the time points tested (Figure $4 \mathrm{E}$ ).

Summarizing, these data indicate that constitutive expression of GFP-UTF1 completely blocked DMSO-induced differentiation of P19CL6 EC cells. In ES cells that constitutively express GFP-UTF1, a subset of cells is observed that is (at least to some extent) delayed in their differentiation or even refractory to differentiation during $\mathrm{EB}$

A

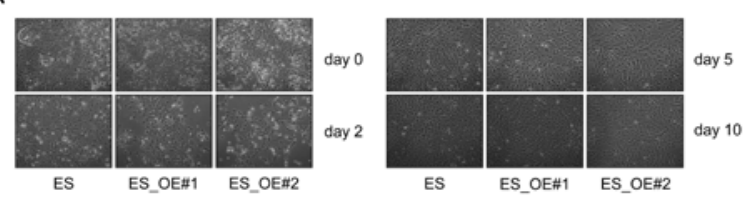

B

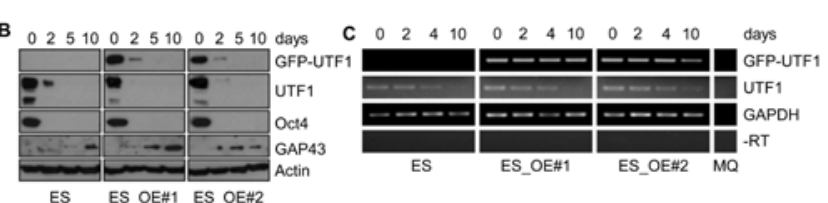

D
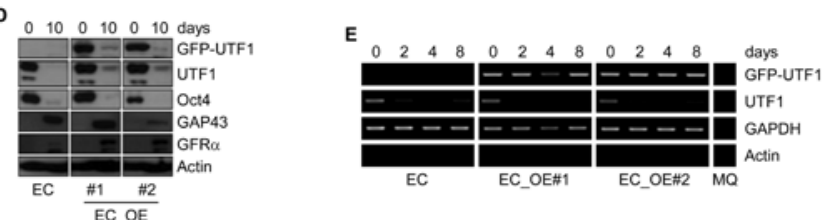

Figure 4: RA differentiation of ES and EC cells overexpressing GFP. UTF1. A) Phase-contrast images of RA-treated (0, 2, 5 and 10 days) of wild type (ES) and GFP-UTF1 overexpressing ES cells (ES_OE\#1 and \#2).

$B$ and $D$ ) Western blots of RA-induced differentiation (0, 2, 5 and 10 days) of wild type (ES and EC) and GFP-UTF1 overexpressing IB10 ES and P19CL6 EC cells (OE\#1 and \#2). Protein lysates were analyzed with antibodies against GFP, UTF1, Oct4, GAP43 (ectoderm) and GFRa (ectoderm). Actin staining was performed as a loading control.

$C$ and E) RT-PCR analysis of UTF1 and GFP-UTF1 expression in wild type (ES and EC) and GFP-UTF1 overexpressing IB10 ES and P19CL6 EC cells (OE\#1 and \#2) during RA treatment. Glyceraldehyde-3-phosphate dehydrogenase (GAPDH) expression was used as a control. In the -RT lanes, reverse transcriptase was omitted from the reverse transcriptase reactions to control for genomic DNA contamination and amplified using GAPDH primers. In the $\mathrm{MQ}$ lane, no template was added to the $\mathrm{PCR}$ reactions. formation. Finally, RA treatment resulted in a rapid down regulation of (GFP)-UTF1 protein, which preceded the down regulation of UTF1 mRNA, strongly suggesting that UTF1 is actively degraded in response to RA.

\section{Discussion}

We previously reported that ES and EC cells with strongly reduced UTF1 levels are defective in their differentiation and that in UTF1depleted ES cells the expression of $>1200$ genes is increased $[21,28]$. As UTF1 is highly expressed in ES and EC cells and rapidly down regulated upon differentiation, ES and EC cell lines expressing GFP-UTF1 were generated to investigate the effect of constitutive UTF1 expression.

ES and EC cells with GFP-UTF1 expression levels similar to endogenous UTF1 levels were selected. Despite the relatively high levels of GFP-UTF1 expression, GFP-UTF1 was exclusively nuclear localized and associated with mitotic chromosomes. Subnuclear fractionation analysis further showed that GFP-UTF1, like endogenous UTF1, was only detected in the ammonium sulphate fraction, the fraction containing tightly chromatin-associated proteins. Finally, the expression of several UTF1 target genes was reduced in GFPUTF1 expressing ES cells, suggesting that GFP-UTF1 repressed the expression of these UTF1 target genes. These observations indicate that GFP-UTF1, although expressed at relatively high levels, displayed properties identical to endogenous UTF1.

Most GFP-UTF1 overexpressing cell lines (3 out of 4 EC lines and both ES lines) displayed increased doubling times. Some variation in the observed proliferation of the different lines might originate from clonal variation as these lines are subclones from the original P19CL6 and IB10 cell lines. Previously, Nishimoto et al. and we reported an increased doubling time of ES cells with reduced UTF1 levels [21,33]. These data suggest that either under- or overexpression of UTF1 has a detrimental effect on the proliferative capacity of ES and EC cells. During EB differentiation, in wt ES cells UTF1 and Oct4 levels were practically not detected after day 4 where in GFP-UTF1 overexpressing ES cells, UTF1 and Oct4 expression was much longer maintained. However, the induction of differentiation markers was not drastically delayed in GFP-UTF1 expressing ES cells, suggesting that a subset of the cells differentiated normally and that another subset remained Oct4 and UTF1 positive. These observations were confirmed in EB sections where expression of GFP-UTF1 and OCT4 overlapped and was not detected in cells expressing differentiation markers like GATA4.

Only a subset of wt and GFP-UTF1 expressing ES cells differentiated in response to DMSO, as relatively high amounts of both UTF1 and Oct4 were detected at all time points. Differentiation markers were induced in wt and GFP-UTF1 expressing cells with similar kinetics indicating that DMSO-induced differentiation was not affected by GFP-UTF1 overexpression. However, differentiation of P19CL6 EC cells was completely blocked by GFP-UTF1 overexpression. P19CL6 cells are a subclone of P19EC cells selected for differentiation towards cardiac muscle in response to DMSO [30]. GATA4 is a transcription factor up regulated during DMSO-induced cardiac differentiation of P19CL6 cells [36]. In a UTF1 ChIP-on-chip experiment, GATA4 was not identified as a direct UTF1 target gene, suggesting that GATA4 is not directly repressed by GFP-UTF1 [28]. P19CL6 cells with reduced UTF1 levels were also completely defective in their ability to differentiate in response to DMSO [21]. This indicates that for P19CL6 cells to properly differentiate, UTF1 levels need to be properly controlled. 
RA readily induced differentiation of both ES and EC cells expressing GFP-UTF1. RA treatment resulted in a rapid silencing of the endogenous UTF1 gene (both mRNA and protein) as well as a drastic reduction GFP-UTF1 protein levels. Remarkably however, RT-PCR analysis indicated that the GFP-UTF1 transgene was still transcribed. RA-induced proteasome activation has been reported in acute myeloid leukemic cells [37] as well as enhanced proteasome activity during ES cell differentiation [38], suggesting that UTF1 might be a target of RA-induced proteosomal degradation. In this study, we show that overexpression of the chromatin-associated protein UTF1 interferes with the differentiation of a subset of ES cells where in EC cells, overexpression of GFP-UTF1 completely blocked differentiation towards cardiac muscle. These data, in combination with our earlier observations that UTF1 KD ES and EC cells displayed extensive differentiation defects, suggest that in order for proper ES or EC cell differentiation, the levels of UTF1 expression need to be within a certain range. An approximate two-fold increase (as in this study) or a ten-fold reduction (as in [21]) in UTF1 levels negatively interferes with ES and EC cell differentiation.

\section{Acknowledgement}

The authors thank Susanne M. Kooistra, Jyothi Thyagabhavan Mony, Rutger A. Gjaltema, Michel Meijer and letje Mantingh-Otter for stimulation discussions and experimental assistance.

\section{References}

1. Murakami M, Ichisaka T, Maeda M, Oshiro N, Hara K, et al. (2004) mTOR is essential for growth and proliferation in early mouse embryos and embryonic stem cells. Mol Cell Biol 24: 6710-6718.

2. Sato N, Meijer L, Skaltsounis L, Greengard P, Brivanlou AH (2004) Maintenance of pluripotency in human and mouse embryonic stem cells through activation of Wnt signaling by a pharmacological GSK-3 specific inhibitor. Nat Med 10: 55-63.

3. Smith AG, Heath JK, Donaldson DD, Wong GG, Moreau J, et al. (1988) Inhibition of pluripotential embryonic stem cell differentiation by purified polypeptides. Nature 336: 688-690.

4. Williams RL, Hilton DJ, Pease S, Willson TA, Stewart CL, et al. (1988) Myeloid leukaemia inhibitory factor maintains the developmental potential of embryonic stem cells. Nature 336: 684-687.

5. Ying QL, Nichols J, Chambers I, Smith A (2003) BMP induction of id proteins suppresses differentiation and sustains embryonic stem cell self-renewal in collaboration with STAT3. Cell 115: 281-292.

6. Ying QL, Wray J, Nichols J, Batlle-Morera L, Doble B, et al. (2008) The ground state of embryonic stem cell self-renewal. Nature 453: 519-523.

7. Nichols J, Zevnik B, Anastassiadis K, Niwa H, Klewe-Nebenius D, et al. (1998) Formation of pluripotent stem cells in the mammalian embryo depends on the POU transcription factor Oct4. Cell 95: 379-391.

8. Chambers I, Colby D, Robertson M, Nichols J, Lee S, et al. (2003) Functional expression cloning of nanog, a pluripotency sustaining factor in embryonic stem cells. Cell 113: 643-655.

9. Mitsui K, Tokuzawa Y, Itoh H, Segawa K, Murakami M, et al. (2003) The homeoprotein nanog is required for maintenance of pluripotency in mouse epiblast and ES cells. Cell 113: 631-642.

10. Avilion AA, Nicolis SK, Pevny LH, Perez L, Vivian N, et al. (2003) Multipotent cell lineages in early mouse development depend on SOX2 function. Genes Dev 17: $126-140$

11. Chi AS, Bernstein BE (2009) Developmental biology. Pluripotent chromatin state. Science 323: 220-221.

12. Gao X, Tate P, Hu P, Tjian R, Skarnes WC, et al. (2008) ES cell pluripotency and germ-layer formation require the SWI/SNF chromatin remodeling component BAF250a. Proc Natl Acad Sci USA 105: 6656-6661.
13. Yan Z, Wang Z, Sharova L, Sharov AA, Ling C, et al. (2008) BAF250Bassociated SWI/SNF chromatin-remodeling complex is required to maintain undifferentiated mouse embryonic stem cells. Stem Cells 26: 1155-1165.

14. Kaji K, Caballero IM, MacLeod R, Nichols J, Wilson VA, et al. (2006) The NuRD component $\mathrm{Mbd} 3$ is required for pluripotency of embryonic stem cells. Nat Cell Biol 8: 285-292.

15. Liang J, Wan M, Zhang Y, Gu P, Xin H, et al. (2008) Nanog and Oct4 associate with unique transcriptional repression complexes in embryonic stem cells. Nat Cell Biol 10: 731-739.

16. Loh YH, Zhang W, Chen X, George J, Ng HH (2007) Jmjd1a and Jmjd2c histone $\mathrm{H} 3$ lys 9 demethylases regulate self-renewal in embryonic stem cells. Genes Dev 21: 2545-2557.

17. Orkin SH, Hochedlinger K (2011) Chromatin connections to pluripotency and cellular reprogramming. Cell 145: 835-850.

18. Meshorer E, Yellajoshula D, George E, Scambler PJ, Brown DT, et al. (2006) Hyperdynamic plasticity of chromatin proteins in pluripotent embryonic stem cells. Dev Cell 10: 105-116.

19. Efroni S, Duttagupta R, Cheng J, Dehghani H, Hoeppner DJ, et al. (2008) Global transcription in pluripotent embryonic stem cells. Cell Stem Cell 2: 437 447.

20. Gaspar-Maia A, Alajem A, Polesso F, Sridharan R, Mason MJ, et al. (2009) Chd1 regulates open chromatin and pluripotency of embryonic stem cells. Nature 460: 863-868.

21. van den Boom V, Kooistra SM, Boesjes M, Geverts B, Houtsmuller AB, et al. (2007) UTF1 is a chromatin-associated protein involved in ES cell differentiation. J Cell Biol 178: 913-924.

22. Lin $\mathrm{CH}$, Yang $\mathrm{CH}$, Chen YR (2012) UTF1 deficiency promotes retinoic acidinduced neuronal differentiation in P19 embryonal carcinoma cells. Int $J$ Biochem Cell Biol 44: 350-357.

23. Okuda A, Fukushima A, Nishimoto M, Orimo A, Yamagishi T, et al. (1998) UTF1, a novel transcriptional coactivator expressed in pluripotent embryonic stem cells and extra-embryonic cells. EMBO J 17: 2019-2032.

24. Chuva de Sousa Lopes SM, van den Driesche S, Carvalho RL, Larsson J, Eggen B, et al. (2005) Altered primordial germ cell migration in the absence of transforming growth factor beta signaling via ALK5. Dev Biol 284: 194-203.

25. van Bragt MP, Roepers-Gajadien HL, Korver CM, Bogerd J, Okuda A, et al. (2008) Expression of the pluripotency marker UTF1 is restricted to a subpopulation of early A spermatogonia in rat testis. Reproduction 136: 33-40

26. Kristensen DM, Nielsen JE, Skakkebaek NE, Graem N, Jacobsen GK, et al. (2008) Presumed pluripotency markers UTF-1 and REX-1 are expressed in human adult testes and germ cell neoplasms. Hum Reprod 23: 775-782.

27. Melone MA, Giuliano M, Squillaro T, Alessio N, Casale F, et al. (2009) Genes involved in regulation of stem cell properties: Studies on their expression in a small cohort of neuroblastoma patients. Cancer Biol Ther 8: 1300-1306.

28. Kooistra SM, van den Boom V, Thummer RP, Johannes F, Wardenaar R, et al. (2010) Undifferentiated embryonic cell transcription factor 1 regulates ESC chromatin organization and gene expression. Stem Cells 28: 1703-1714.

29. Kooistra SM, Thummer RP, Eggen BJ (2009) Characterization of human UTF1, a chromatin-associated protein with repressor activity expressed in pluripotent cells. Stem Cell Res 2: 211-218.

30. Habara-Ohkubo A (1996) Differentiation of beating cardiac muscle cells from a derivative of P19 embryonal carcinoma cells. Cell Struct Funct 21: 101-110.

31. Citterio E, Papait R, Nicassio F, Vecchi M, Gomiero P, et al. (2004) Np95 is a histone-binding protein endowed with ubiquitin ligase activity. Mol Cell Biol 24 2526-2535.

32. Livak KJ, Schmittgen TD (2001) Analysis of relative gene expression data using real-time quantitative PCR and the 2(-delta delta $\mathrm{C}(\mathrm{T})$ ) method. Methods 25: 402-408.

33. Nishimoto M, Miyagi S, Yamagishi T, Sakaguchi T, Niwa H, et al. (2005) Oct$3 / 4$ maintains the proliferative embryonic stem cell state via specific binding to 
Citation: Thummer RP, Drenth-Diephuis LJ, Eggen BJL (2012) Constitutive GFP-UTF1 Expression Interferes with ES and EC Cell Differentiation. J Stem Cell Res Ther 2:127. doi:10.4172/2157-7633.1000127

Page 7 of 7

a variant octamer sequence in the regulatory region of the UTF1 locus. Mol Cell Biol 25: 5084-5094

34. Dani C, Chambers I, Johnstone S, Robertson M, Ebrahimi B, et al. (1998) Paracrine induction of stem cell renewal by LIF-deficient cells: A new ES cell regulatory pathway. Dev Biol 203: 149-162.

35. McBurney MW, Jones-Villeneuve EM, Edwards MK, Anderson PJ (1982) Control of muscle and neuronal differentiation in a cultured embryonal carcinoma cell line. Nature 299: 165-167.

36. Snyder M, Huang XY, Zhang JJ (2010) Stat3 directly controls the expression of
Tbx5, Nkx2.5, and GATA4 and is essential for cardiomyocyte differentiation of P19CL6 cells. J Biol Chem 285: 23639-23646.

37. Fang Y, Zhou X, Lin M, Ying M, Luo P, et al. (2011) Inhibition of all-transretinoic acid-induced proteasome activation potentiates the differentiating effect of retinoid in acute myeloid leukemia cells. Mol Carcinog 50: 24-35.

38. Hernebring M, Brolen G, Aguilaniu H, Semb H, Nystrom T (2006) Elimination of damaged proteins during differentiation of embryonic stem cells. Proc Nat Acad Sci USA 103: 7700-7705. 